\title{
A Suggested Fellowship of Spine in Sports Medicine
}

\author{
Pardis Noormohammadpour ${ }^{1,2}$ and Afifeh Khosravi (id) ${ }^{1, *}$ \\ ${ }^{1}$ Sports Medicine Research Center, Neuroscience Institute, Tehran University of Medical Sciences, Tehran, Iran \\ ${ }^{2}$ Department of Sports and Exercise Medicine, School of Medicine, Tehran University of Medical Sciences, Tehran, Iran \\ Corresponding author: Sports Medicine Research Center, Neuroscience Institute, Tehran University of Medical Sciences, Tehran, Iran. Tel: +98-9214543747, Email: \\ nursingkhosravi@gmail.com
}

Received 2019 December 15; Revised 2020 March 16; Accepted 2020 March 22.

Keywords: Spine, Sports, Fellowship

While sports medicine is defined as taking care of recreational and professional athletes, many of the actual patients in sports medicine clinics refer due to musculoskeletal conditions, including peripheral joint, soft tissue, and spinal pains. The sports medicine program for medical graduates is usually structured as a subspecialty or a distinct specialty program (1).

In the United States, the debate is over whether this sports medicine scholarship can prepare graduates to meet the growing need for skeletal and muscular care. Sports medicine is presented as a subspecialty fellowship in the United States. However, there is an ongoing debate in the United States about this fellowship. It is debated that if the sports medicine fellowship can prepare graduates to meet the growing need for skeletal and muscular care. The graduates of this fellowship are usually trained for peripheral joint and soft tissue care, and they lack education and skills for interventional spine care. Moreover, there are some unaccredited spine and sports and musculoskeletal and spine fellowships in response to the need for a training program to address both peripheral joint and spinal conditions (2).

Sports medicine specialists are trained for peripheral joint and soft tissue care. The lack of education on spine care is also the case in countries with sports medicine as a distinct specialty, such as the UK, Turkey, and Australia.

We gathered all curriculums of Sports Medicine, Sports and Spine, and Spine residency and fellowship programs, and then selected spine-related topics. We believe this list can include all needed educational goals for a complimentary "spine in sports" fellowship for graduates of sports medicine as a distinct specialty (supplementary file Appendices 1 ). The graduates of sports medicine as a distinct specialty are trained for two to four 4 years to address the pe- ripheral joint and muscle problems. Thus, the proposed "spine in sports" fellowship educational goals would not include peripheral joint care, and it would be a short (oneyear) complementary program.

\section{Supplementary Material}

Supplementary material(s) is available here [To read supplementary materials, please refer to the journal website and open PDF/HTML].

\section{Footnotes}

Conflict of Interests: None of the authors mentioned conflicts of interest.

Funding/Support: None declared by the author.

\section{References}

1. Pigozzi F. Specialisation in sports medicine: the state of the Sport Medicine Specialty Training Core Curriculum in the European Union. Br J Sports Med. 2009;43(14):1085-7. doi: 10.1136/bjsm.2008.055350. [PubMed: 19282304].

2. Mayer EK, Ihm JM, Sibell DM, Press JM, Kennedy DJ. ACGME sports, ACGME pain, or non-ACGME sports and spine: which is the ideal fellowship training for PM\&R physicians interested in musculoskeletal medicine? PM R. 2013;5(8):718-23. discussion 723-5. doi: 10.1016/j.pmrj.2013.07.004. [PubMed: 23953018]. 